\title{
Design and Implementation of Training Management Software for General Simulation Training System
}

\author{
Wei Xiang ${ }^{1, *}$, Sun Chunsheng ${ }^{1}$, and Dong Yanhong ${ }^{1}$ \\ ${ }^{1}$ Navy Submarine Academy, Qingdao 266199, Shandong Province, China
}

\begin{abstract}
The common simulation training system realizes the common design of hardware and software, and also increases the difficulty of group training management. In order to solve the problem of flexible group training of multiple training modes for general simulation training system of a certain type of naval combat system, this paper puts forward a formal description method of group training resources and group training programs based on an in-depth analysis of training management requirements. Design and implementation of a general simulation training system training management software.
\end{abstract}

\section{Introduction}

With the continuous development of simulation technology, the development of general simulator or simulation training system suitable for specific fields has played an important role in personnel training, playing a significant economic and social benefits ${ }^{[1-3]}$. Universal simulation training system to achieve a common hardware and software design ${ }^{[4-8]}$, but also increased the difficulty of group training management.

The authors recently developed a general warship combat simulation training system, including four training platforms and three kinds of simulation software, which can realize three types of training modes, such as single professional multi-person training, multiprofessional multi-person training, and whole system cooperative training.

In the process of development, a flexible group training management software based on group training resources and group training programs was designed and implemented. Practice has proved that the software can better solve the multi-mode flexible group training problem of general simulation training system.

\section{A generic simulation training system group training resources}

\subsection{Hardware group training resources}

Hardware group training resources mainly refers to various types of training platform used to meet the group training staff and trainee use. According to the structure and function of each training station, it can be divided into four types: universal teaching console, general virtual simulation console, general analog standard console and special analog console. Among them, the general virtual simulation console and general analog standard control console can run different professional equipment simulation software, so as to achieve different professional training functions.

Universal teaching console is used to run all kinds of teaching control software, from the control cabinet, mainframe, monitors, LCD electronic signage, keyboard and mouse, voice Mike interface and so on. General virtual simulation console used to run the professional equipment of the virtual simulation software, the control cabinet, mainframe, monitors, LCD electronic signage, keyboard and mouse components. The general analog standard console is used to realize the display and console simulation function of the electronic equipment, which is composed of cabinet, display, host, control panel, LCD electronic signboard and so on.The special analog console is mainly used to simulate the nonstandard professional equipment. The special analog cabinet has no unified standard structure, which is consistent with the corresponding professional equipment's exterior structure.

\subsection{Software group training resources}

Software group training resources mainly refers to the various types of software running in the hardware platform. According to the function can be divided into teaching and control software, virtual simulation software, simulation software, three categories.

The teaching and control software is installed in the host of the general education console. It is used for online management of whole system equipment, training and setting of roles and functions, training director control, training operation process monitoring, training record and evaluation, training place audio-video monitoring and other functions. The virtual simulation software is installed and run on the host of the general virtual simulation platform, and simulates the appearance and operation flow of the corresponding

\footnotetext{
*Corresponding author: weix826@aliyun.com
} 
equipment through software, and realizes the simulation of the equipment function and performance through the simulation model. The simulation software is installed and run on a common analog standard control console and a dedicated analog control console. The simulation signal is received according to the operation signal of the analog table component, and is calculated according to the simulation model. The display part of the analog table is driven to display the corresponding calculation result.

\section{Group training management needs analysis}

\subsection{Group training mode}

The common simulation training system for a certain type of warship operation system can realize three training modes including single-profession simultaneous multi-person training, multi-professional multi-person simultaneous training and system-wide collaborative training.

When organizing a single professional multi-person training at the same time, you need to configure and automatically start the virtual simulation platform, analog standard control console or the corresponding professional simulation software on the dedicated analog control console through the group training management software so as to achieve the same professional multiperson simultaneous training, A certain type of general combat training system for combat training system can achieve up to 36 cadets at the same time a professional training. In the organization of multi-professional and multi-people training at the same time, according to training needs through group training management software for the corresponding configuration, you can achieve any combination of multiple professional training at the same time. In the organization of systemwide collaborative training, all the professional equipment simulation software of the combat system needs to be activated through the configuration of the group training management software, and the information interaction among various professional simulation softwares is realized through the network so as to realize the system-wide collaborative training function of the combat system.

\subsection{Group training resources allocation principles}

In order to achieve different group training mode, it is necessary to assign corresponding teaching control software and simulation software to each training station through group training management software. Group training resource allocation refers to the group training needs to achieve the system hardware and software resource allocation process. The following principles need to be followed in the group training resource allocation process of the general simulation training system of a certain type of naval combat system.
1) The principle of pertinence. Group training resource allocation should be in accordance with the requirements of group training mode for each station assigned the appropriate software resources.

2) Integrity principle. When conducting system-wide collaborative training, you need to deploy a full suite of specialized equipment simulation software for your combat system.

3) The principle of feasibility. The software that each type of hardware platform can run is limited, so the resource allocation needs to consider the feasibility of the hardware platform. Universal teaching station can be assigned to run a variety of teaching control software, universal virtual simulation platform can be assigned to run a variety of virtual simulation software, universal analog standard console can be assigned to run a variety of standard console simulation software, dedicated analog console can be assigned to run the corresponding professional equipment simulation software.

\section{Sets of training information description method}

\subsection{Group training resource description}

Group training resources include system hardware resources and software resources. In order to implement flexible training management functions, all training resources need to be formally described. The definition of group training resource description is given below.

Definition 1: SimSub ships SimSub is made up of $r$ model ships Sub, with independent functions and features that can be run alone.

$$
\operatorname{SimSub}=\left\{\operatorname{Sub}_{i}, i=1,2, \ldots, r\right\}
$$

Definition 2: A single type of sub-ship Sub is composed of s different professional analog equipment positions ProfessionE, in accordance with certain combinations to achieve naval cooperative training.

$$
S u b=\left\{\text { Profession }_{i}, i=1,2, \ldots, s\right\}
$$

Definition 3: Single professional simulation equipment position ProfessionE is simulated by SimWay, equipment name EName, which type Type, equipment program ESoft.

$$
\text { ProfessionE }=\{\text { SimWay,Ename,Type,ESoft }
$$

According to the definition 1 design different types of ship configuration data, according to the definition 2 and the definition of 3 different types of equipment configuration data design. Finally, the ship configuration information and equipment configuration information generated by the combination of platform information description file.

\subsection{Group training program description}

Group training program description includes group training mode information, group training mode and 
equipment correspondence information as well as training equipment attribute information.

Definition 4: Group training mode The OrgTModel is composed of three training modes TModel, each training mode has an independent training function.

$$
\text { OrgTModel }=\left\{\text { TModel }_{i}, i=1,2,3\right\}
$$

Definition 5: Each training mode TModel is composed of $n$ training equipment TE.

$$
\text { TModel }=\left\{T E_{i}, i=1,2, \cdots, n\right\}
$$

Definition 6: Each training equipment $\mathrm{TE}$ is composed of the host name of the simulator, SHost, the electronic nameplate access server name CName, the equipment name EName, the type of the equipment, and the equipment program ESoft.

$$
T E=\{\text { SHost }, \text { Cname,Ename }, \text { Type }, \text { ESoft }
$$

\section{Training management software design}

The main functions of the training management software include: reading the training station information configuration file, realizing the flexible configuration of the hardware and software resources, monitoring the network status in real time, and sending the station configuration information. The main interface of the software is shown in Fig. 1.

Fig.1 Training Management Software Interface

This article is based on object-oriented technology design training management software, using $\mathrm{C}++$ programming.

(1) The left tree view class CLeftView

This class is mainly used to complete the read, record, display group training resource information and hardware and software resource configuration operations, the main methods are ReadIniFile and DisposeE.

ReadIniFile method is used to read the station information configuration file and display the analog device. The input parameter is the location information profile path. The output parameter is an array of structures for all station information.

DisposeE method to configure the simulation device. Input parameters for analog devices and their related information. The output is a Boolean type that identifies whether the configuration was successful.

(2) List box view class CFormViewEx

This class is used to complete the reading, recording, display each group training configuration file information and send analog device information, the main method is ReadIniFile, SndOrder.

ReadIniFile method used to read the group training configuration file and display it in the interface. Input parameters for the group training configuration file path.
The output parameter is an array of structures that record all the training configuration files.

(3) The main form of class CMainFrame

This class is used to complete the network connection and its detection, display station information and each group training configuration file information, the main method is HandleNetWork. This method realizes the network connection, the network receives the data.

\section{Conclusion}

In this paper, the software of group training management is designed and realized,which was based on the formal description method of group training resources and group training programs. The practice has proved that the software can solve the flexible group training problem of the general simulation training system. At the same time, the group training management solution is also applicable to other similar general simulation training systems.

\section{References}

1. CHEN Lei, WANG Song, ZHANG Feng. Demand Analysis of Universal Simulator [J] .Computer Science and Modernization, 2012 (8): 166-169.

2. Fan Cheng, Zhou An-dong, Yang Lu-gang.A universal simulator LCD display scheme design [J]. Computer and Digital Engineering, 2010,38 (5): 163-165.

3. ZOU Xian-Yang, HU Wei-Biao, WANG Qi.Design of Embedded System Radar Electronic Interference Simulator [J]. Process Automation Instrumentation, 2012,33 (6): 70-72

4. Wang Q, Hao M A, Rong X, et al. Design and Implementation of the Directing and Adjusting Control of Environment in a Common Simulation Training System[J]. Fire Control \& Command Control, 2012.

5. Yang J. Design of Artillery Fight Training Simulation Directing and Adjusting System Based on Information System[J]. Command Control \& Simulation, 2011.

6. Guangzhou. Design of Directing \& Adjusting Frame in Combat Simulation Training System[J]. Ship Electronic Engineering, 2008.

7. Wang X, Wang W, Liu Q, et al. Design and Realization of Naval Air Combat Simulation System's Director Platform[J]. System Simulation Technology, 2010.

8. Sheng D H, Qiu X G. Design and Realization of Directing and Adjusting Federate Member and Its Surroundings in Fight Simulation[J]. Computer Simulation, 2004, 21(10):14-17. 